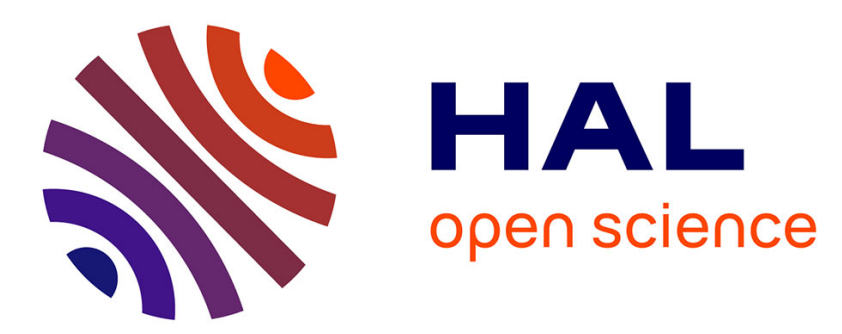

\title{
Steady state forced response of a mechanical oscillator with combined parametric excitation and clearance type non-linearity
}

G.W. Blankenship, A. Kahraman

\section{- To cite this version:}

G.W. Blankenship, A. Kahraman. Steady state forced response of a mechanical oscillator with combined parametric excitation and clearance type non-linearity. Journal of Sound and Vibration, 1995, 185 (5), pp.743 - 765. 10.1006/jsvi.1995.0416 . hal-01389915

\section{HAL Id: hal-01389915 \\ https://hal.science/hal-01389915}

Submitted on 30 Oct 2016

HAL is a multi-disciplinary open access archive for the deposit and dissemination of scientific research documents, whether they are published or not. The documents may come from teaching and research institutions in France or abroad, or from public or private research centers.
L'archive ouverte pluridisciplinaire HAL, est destinée au dépôt et à la diffusion de documents scientifiques de niveau recherche, publiés ou non, émanant des établissements d'enseignement et de recherche français ou étrangers, des laboratoires publics ou privés.

\section{(c)(1)}

Distributed under a Creative Commons Attribution| 4.0 International License 


\title{
STEADY STATE FOR CED RESPONSE OF A MECHANICAL OSCILLATOR WITH COMBINED PARAMETRIC EXCITATION AND CLEARANCE TYPE NON-LINEARITY
}

\author{
G. W. Blankenship and A. Kahraman \\ General Motors Corporation, Powertrain Division, 37350 Ecorse Road, Romulus, \\ Michigan 48174-1376 U.S.A.
}

\begin{abstract}
A mechanical system exhibiting combined parametric excitation and clearance type non-linearity is examined analytically and experimentally in an effort to explain complex behavior that is commonly observed in the steady state forced response of rotating machines. The specific case of a preloaded mechanical oscillator having a periodically time-varying stiffness function and subject to a symmetric backlash condition is considered. A generalized solution methodology is proposed based on the harmonic balance method. The resulting non-linear algebraic equations are solved by using a direct Newton-Raphson technique, in which a closed form Jacobian matrix is computed using frequency domain methods. Analytical solutions are validated by comparison with numerical integration results and experimental measurements obtained from a gear dynamics test rig.
\end{abstract}

\section{INTRODUCTION}

In the study of rotating machinery dynamics, of primary interest is the prediction and measurement of the steady state forced response as excited by internal system components such as gears, rolling element bearings, turbines, pumps, sprags, clutches and the like. Such systems are inherently non-linear with temporally and spatially varying system parameters which arise due to periodically changing contact regimes and separations due to clearances or backlash. For instance, in a spur gear pair, the gear mesh stiffness varies periodically with the angular positions of the gears as the number of tooth pairs in contact alternates between $n$ and $n+1$, where $n$ is the integer part of the profile contact ratio. Similarly, the radial stiffness of a ball or roller bearing is time-varying due to fluctuations in the number of rolling elements in contact as the shafts rotate. In both examples, a clearance is introduced into the system in the form of gear backlash or a radial gap to ease the assembly and ensure proper operation.

In the course of the authors' experimental investigations with mechanical power transmission systems, several phenomena have been observed in the forced response of such systems which are yet to be explained analytically; these include but are not limited to multiple coexisting limit cycles, modulation sidebands in narrow band spectra, sub- and superharmonic motions and aperiodic or apparent chaotic behavior. In an attempt to develop a fundamental understanding of the physical mechanisms which give rise to such complex behavior, a governing equation is introduced based on the simple mechanical 
oscillator of unit mass shown in Figure 1:

$$
\begin{gathered}
\ddot{x}+2 \zeta \dot{x}+\psi[\theta(\tau)] g[x(\tau)]=f(\tau), \\
\psi(\theta)=w_{1}+\sum_{k=1}^{K}\left[w_{2 k} \cos (k \theta)+w_{2 k+1} \sin (k \theta)\right], \\
g[x(\tau)]= \begin{cases}x(\tau)-1, & x(\tau)>1, \\
0, & |x(\tau)| \leqslant 1, \\
x(\tau)+1, & x(\tau)<-1 .\end{cases}
\end{gathered}
$$

Here, $(\cdot)$ denotes differentiation with respect to time $\tau, \zeta$ is an equivalent system damping ratio, $f(\tau)$ is an external forcing function, $\psi[\theta(\tau)]=\psi[\theta(\tau)+2 \pi]$ is a spatially periodic stiffness function and $g[x(\tau)]$ is a non-linear restoring function. In general, $\psi(\theta)$ is assumed to be positive definite with $w_{1}=1$ and can therefore be expressed in terms of the Fourier series given by equation (1b). The restoring function $g[x(\tau)]$ may describe softening or hardening characteristics as well as clearance type behavior. In this study, $g[x(\tau)]$ is assumed to be a symmetric, piecewise-linear clearance or backlash function as defined by equation (1c). The external forcing function $f(\tau)$ may take on many different forms depending upon the system configuration, but is usually assumed to be periodic in $\theta$ and can, therefore, be represented as the Fourier series

$$
f(\theta)=f_{1}+\sum_{l=1}^{L}\left[f_{2 l} \cos (l \theta)+f_{2 l+1} \sin (l \theta)\right] .
$$

The system rotation angle $\theta(\tau)$ is given by $\theta(\tau)=\Lambda \tau+\beta x(\tau)$, where $\Lambda$ is a time-invariant mean angular velocity. The term $\beta x(\tau)$ represents a linear variation in $\theta(\tau)$ with $x(\tau)$. Hence, $\psi(\theta)$ and $f(\theta)$ are both exponentially modulated by $x(\tau)$ and $\beta$ is the so-called angle modulation depth [1].

\subsection{LITERATURE REVIEW}

Previous studies in the literature have focused almost entirely on two limiting cases of equation (1). By far the most studied form of equation (1) is the linear system obtained by neglecting the clearance non-linearity such that $g[x(\tau)]=x(\tau)$. This leads to a form of the Hill or Mathieu equation [2]. In most studies, sinusoidal or piecewise linear variations of the system parameters are assumed and, typically, only the initial value problem is solved



Figure 1. The physical system: a mechanical oscillator of unit mass. 
with an emphasis on dynamic stability and parametric behavior of the system [2]. A general solution methodology for the steady state forced response of systems subject to combined parametric and forcing excitations was presented by Hsu and Cheng [3] in terms of the fundamental matrix of the homogeneous system. The harmonic balance approach has also been employed by Blankenship and Singh [1] to explain the presence of modulation sidebands in machinery spectra.

The other limiting case of equation (1) considers a piecewise linear oscillator with external excitation and time-invariant stiffness such that $\psi[\theta(\tau)]=1$. Such systems have been shown to exhibit an entire spectrum of nonlinear phenomena such as the dependence of steady state solutions to initial conditions, multiple coexisting solutions as well as subharmonic and chaotic motions. Due to the strong non-linearity introduced by $g[x(\tau)]$, standard perturbation solution techniques are not suitable [4]. The viable solution techniques may be divided into four categories. Piecewise linear solution techniques, such as those employed by Shaw and Holmes [5] and Natsiavas [6], seek closed form linear solutions corresponding to each segment of $g[x(\tau)]$. The resulting solutions must be precisely matched at the instants when impact occurs. This procedure may be quite difficult to implement, especially with impacts that are not equally spaced. Single and multiple term harmonic balance (HBM) approaches have been employed effectively to obtain the steady state forced response by several investigators including Tomlinson and Lam [7], Kahraman and Singh [4], Pfeiffer and Fritzer [8], Choi and Noah [9], Kim and Noah [10] and Lau and Zhang [11]. One limitation of HBM is that the effects of initial conditions on the solution cannot be considered. A third method introduced by Pfeiffer and Kunert [12] uses a stochastic model applied to the well-known Fokker-Planck equation. Finally, direct numerical integration has been widely employed to study systems with clearance type non-linearities; examples include the investigations by Mahfouz and Badrakhan [13] and Kahraman [14]. Although very valuable as a tool to validate other analytical methods, numerical integration is not practical due to the computational expense required to obtain steady state solutions, especially for lightly damped systems.

The main focus of this study is the steady state forced response of preloaded systems exhibiting combined parametric excitation and clearance type nonlinearities. Although one limiting version of equation (1) having an external alternating forcing term was solved numerically by Kahraman and Singh [15] and Sato et al. [16], a generalized analytical treatment of equation (1) is yet to be developed. Since steady state solutions are of interest in this study, a multiple term HBM approach is employed. The resulting nonlinear algebraic equations are solved by using a direct Newton-Raphson technique where a closed form Jacobian matrix is computed using a frequency domain method similar to that of Kim and Noah [10]. Stability issues are examined using standard perturbation techniques along with well known results from Floquet theory [2].

Experimental studies having as their aim the demonstration of subharmonic and chaotic motions in mechanical systems with clearances have concentrated mostly on small laboratory bench-top set-ups which employ relatively simple non-linear elements. Examples include a beam subjected to a rigid support with clearance [17-19] or an impacting pendulum $[20,21]$. Typically, the apparatus and system parameters are designed such that simple time domain data acquisition and analysis are possible. In this study, a very practical and considerably more complicated engineering application, namely, a precision spur gear pair with backlash and time-varying mesh stiffness characteristics, is used to demonstrate that the proposed governing equation (1) is indeed pertinent to the study of rotating machinery dynamics. Experimental measurements are used clearly to validate the proposed analytical model with an emphasis on describing jump discontinuities in steady state forced response curves. 


\subsection{SCOPE}

A generalized solution methodology is proposed to obtain the steady state forced response of equation (1) for the special case $\beta=0$ such that $\theta(\tau)=\Lambda \tau$. Analytical solutions are compared with numerical integration results and experimental measurements obtained from a gear dynamics test rig. The main emphasis is to obtain analytical solutions which adequately describe measured forced response phenomena in the fundamental and superharmonic resonance regimes. An approximate harmonic solution is obtained by using describing functions in order to arrive at mathematical conditions for the occurrence of non-linear behavior.

\section{GENERAL SOLUTION BY HBM}

The steady state solution $x(\tau)=x(\tau+T)$ of equation (1) is assumed to be periodic in $\tau$ with fundamental period $T=2 \pi / \Lambda$. Accordingly, $g[x(\tau)]=g(\tau)=g(\tau+T)$ must also be periodic in $\tau$ with the same period. Therefore, both functions can be expressed in terms of Fourier series [10]:

$$
\begin{aligned}
& x(\tau)=u_{1}+\sum_{r=1}^{R}\left[u_{2 r} \cos (r \theta)+u_{2 r+1} \sin (r \theta)\right], \\
& g(\tau)=v_{1}+\sum_{r=1}^{R}\left[v_{2 r} \cos (r \theta)+v_{2 r+1} \sin (r \theta)\right] .
\end{aligned}
$$

Equation (2) is substituted into the governing equation (1) and harmonic balance is enforced by collecting even and odd terms of like frequency to form the vector equation $\mathbf{S}=\mathbf{0}$, where the $(2 R+1)$ elements of $\mathbf{S}$ are given by

$$
\begin{gathered}
S_{1}=v_{1}+\frac{1}{2} \sum_{i=1}^{R}\left[v_{2 i} w_{2 i}+v_{2 i+1} w_{2 i+1}\right]-f_{1}, \\
S_{2 r}=-(r \Lambda)^{2} u_{2 r}+2 r \Lambda \zeta u_{2 r+1}+v_{1} w_{2 r}+v_{2 r}+\frac{1}{2} \sum_{i=1}^{R} v_{2 i}\left[w_{2(i-r)}+w_{2(i+r)}+w_{2(r-i)}\right] \\
+\frac{1}{2} \sum_{i=1}^{R} v_{2 i+1}\left[w_{2(i-r)+1}+w_{2(i+r)+1}-w_{2(r-i)+1}\right]-f_{2 r}, \quad r \in[1, R] \\
S_{2 r+1}=-(r \Lambda)^{2} u_{2 r+1}-2 r \Lambda \zeta u_{2 r}+v_{1} w_{2 r+1}+v_{2 r+1}+\frac{1}{2} \sum_{i=1}^{R} v_{2 i}\left[-w_{2(i-r)+1}+w_{2(i+r)+1}\right. \\
\left.+w_{2(r-i)+1}\right]+\frac{1}{2} \sum_{i=1}^{R} v_{2 i+1}\left[w_{2(i-r)}-w_{2(i+r)}+w_{2(r-i)}\right]-f_{2 r+1}, \quad r \in[1, R] .
\end{gathered}
$$

In order to solve $\mathbf{S}=\mathbf{0}$, it is first necessary to express the $v_{i}$ in terms of the $u_{i}$. This is readily accomplished in the frequency domain by making use of the discrete Fourier transform 
(DFT). The values of $x(\tau)$ and $g(\tau)$ at the discrete time $\tau=n h$ are

$$
\begin{gathered}
x_{n}=u_{1}+\sum_{r=1}^{R}\left[u_{2 r} \cos (2 \pi r n / N)+u_{2 r+1} \sin (2 \pi r n / N)\right], \quad n \in[0, N-1], \\
g_{n}=\left\{\begin{array}{ll}
x_{n}-1, & x_{n}>1, \\
0, & \left|x_{n}\right| \leqslant 1, \\
x_{n}+1, & x_{n}<-1,
\end{array} \quad n \in[0, N-1],\right.
\end{gathered}
$$

respectively, where $h=2 \pi /(N \Lambda)$ and $N \geqslant 2 R$ to avoid aliasing errors [22]. The Fourier coefficients of $g(\tau)$ are determined by taking the inverse DFT equation (4b)

$$
\begin{aligned}
& v_{1}=\frac{1}{N} \sum_{n=0}^{N-1} g_{n}, \quad v_{2 r}=\frac{2}{N} \sum_{n=0}^{N-1} g_{n} \cos (2 \pi r n / N), \\
& v_{2 r+1}=\frac{2}{N} \sum_{n=0}^{N-1} g_{n} \sin (2 \pi r n / N), \quad r \in[1, R] .
\end{aligned}
$$

The above $v_{i}$ are then substituted into equation (3) and the solution vector $\mathbf{u}=$ $\left[\begin{array}{llllll}u_{1} & u_{2} & u_{3} & \cdots & u_{2 R} & u_{2 R+1}\end{array}\right]^{\mathrm{T}}$ is determined by employing the Newton-Raphson procedure [23]:

$$
\mathbf{u}^{(m)}=\mathbf{u}^{(m-1)}-\left[\mathbf{J}^{-1}\right]^{(m-1)} \mathbf{S}^{(m-1)} .
$$

Here, $\mathbf{u}^{(m)}$ is the $m$ th iterative solution based on the previous $(m-1)$ th guess and $\mathbf{J}^{-1}$ is the inverse of the Jacobian matrix, the elements of which are defined in Appendix A. The iteration procedure described by equation (6) is repeated until the vector norm of $\mathbf{S}^{(m)}$ is below a specified tolerance. The initial guess $\mathbf{u}^{(0)}$ dictates the number of iterations required for convergence as well as the particular steady state solution found in multiple solution regimes.

\subsection{STABILITY OF STEADY STATE SOLUTION}

The stability of a given periodic solution $x(\tau)$ is determined by examining the stability of the perturbed solution $x(\tau)+\Delta x(\tau)$, using Floquet theory. The variational equation for the perturbation $\Delta x(\tau)$ is given by

$$
\Delta \ddot{x}+2 \zeta \Delta \dot{x}+\psi(\tau) \phi(\tau) \Delta x(\tau)=0,
$$

where $\phi(\tau)$ is a discontinuous separation function

$$
\phi(\tau)= \begin{cases}1, & |x(\tau)|>1, \\ 0, & |x(\tau)| \leqslant 1,\end{cases}
$$

that is consistent with the discrete formulation of equation (A2j) given in Appendix A. Equation (9a) can be written in the state-space form $\dot{\mathbf{z}}(\tau)=\mathbf{G}(\tau) \mathbf{z}(\tau)$, where $\mathbf{z}(\tau)=$ $[\Delta x(\tau) \Delta \dot{x}(\tau)]^{\mathrm{T}}$ is the state vector and $\mathbf{G}(\tau)=\mathbf{G}(\tau+T)$ is the periodic transition matrix given by

$$
\mathbf{G}(\tau)=\left[\begin{array}{cc}
0 & 1 \\
-\psi(\tau) \phi(\tau) & -2 \zeta
\end{array}\right] .
$$


The stability of the perturbed solution and consequently the stability of the corresponding system solution $x(\tau)$ are determined by examining the eigenvalues of the monodromy matrix $\mathbf{M}=\mathbf{Z}(T)$, which is obtained by solving the homogeneous matrix equation $\dot{\mathbf{Z}}(\tau)=\mathbf{G}(\tau) \mathbf{Z}(\tau)$ given initial condition $\mathbf{Z}(0)=\mathbf{I}_{2}$. Here, $\mathbf{I}_{2}$ is the $2 \times 2$ identity matrix. One method to compute $\mathbf{M}$ is by direct numerical integration of the above equation by constructing $\psi(\tau), x(\tau)$, and hence $\phi(\tau)$, from the previously known Fourier coefficients $u_{i}$ and $v_{i}$. A more efficient method to compute $\mathbf{M}$ has been developed by $\mathrm{Hsu}$ and Cheng [3]. The state matrix $\mathbf{G}(\tau)$ is approximated as a series of step functions $\mathbf{G}_{n}$ at $N$ discrete time intervals $\tau=n h$, according to

$$
\mathbf{G}_{n}=\frac{1}{h} \int_{(n-1) h}^{n h} \mathbf{G}(\tau) \mathrm{d} \tau, \quad n \in[0, N-1]
$$

An approximate value of $\mathbf{M}$ is obtained from the expression

$$
\mathbf{M} \cong \prod_{n=0}^{N-1}\left[\mathbf{I}_{2}+\sum_{m=1}^{M}\left(\frac{h \mathbf{G}_{n}^{m}}{m !}\right)\right]
$$

where $M$ is the number of terms in the approximation for the matrix exponential. This method is easily implemented in the present approach, as $\mathbf{M}$ can be readily constructed from the previously available DFT results. The complex eigenvalues $\lambda_{1}$ and $\lambda_{2}$ of $\mathbf{M}$ correspond to the so-called Floquet multipliers which must satisfy the relation $\lambda_{1} \lambda_{2}=\exp (-2 \zeta T)$. The solution $x(\tau)$ is clearly unstable if the modulus of either $\lambda_{1}$ or $\lambda_{2}$ is greater than unity. Bifurcations may also be classified by observing the behavior of the Floquet multipliers as they leave the unit circle [24]. For instance, a saddle node bifurcation, which corresponds to the merger of two periodic solutions, occurs when one multiplier leaves the unit circle at +1 while the other remains inside. A flip bifurcation, which corresponds to the transition from an unstable periodic solution to a stable periodic solution having twice the period, occurs when one multiplier leaves the unit circle at -1 while the other remains inside. Finally, a stable solution will become unstable and doubly periodic whenever a complex conjugate pair leaves the unit circle.

\subsection{HARMONIC SOLUTION}

In an attempt to obtain various closed form expressions which can be used approximately to characterize the system, a single term harmonic balance approach is employed by using describing functions to account for any separations allowed by $g[x(\tau)]$. The stiffness and forcing functions are both assumed to be harmonic at frequency $\kappa \Lambda$, which yields the following reduced form of the governing equation

$$
\ddot{x}(\tau)+2 \zeta \dot{x}(\tau)+\left\{1+w_{a} \sin [\kappa \theta(\tau)]\right\} g[x(\tau)]=f_{m}+f_{a} \sin [\kappa \theta(\tau)+\Gamma] .
$$

Here, subscripts $m$ and $a$ refer, respectively, to the mean and alternating components of the stiffness and forcing functions with $w_{m}=w_{1}=1, w_{a}=\sqrt{w_{2 \kappa}^{2}+w_{2 \kappa+1}^{2}}<1, f_{m}=f_{1}$, $f_{a}=\sqrt{f_{2 \kappa}^{2}+f_{2 \kappa+1}^{2}}$ and relative phase $\Gamma=\tan ^{-1}\left(f_{2 \kappa+1} / f_{2 \kappa}\right)-\tan ^{-1}\left(w_{2 \kappa+1} / w_{2 \kappa}\right)$. The steady state solution is assumed to be purely harmonic:

$$
x(\tau)=u_{m}+u_{a} \sin (\kappa \theta+\alpha),
$$



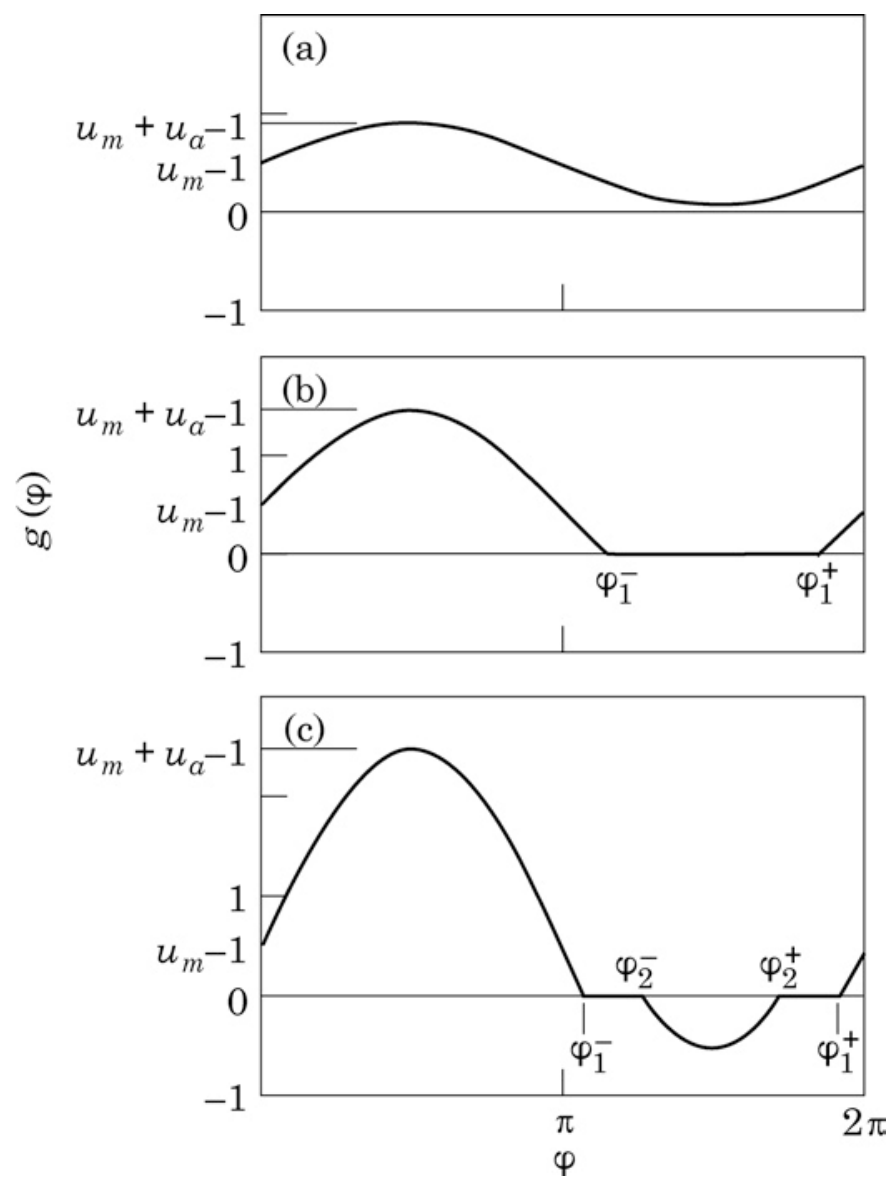

Figure 2. Non-linear restoring function $g(\varphi)$ for (a) no-impact $(N I)$, (b) single sided impact $(S S I)$ and (c) double sided impact regimes $(D S I)$.

where amplitudes $u_{m}$ and $u_{a}$ and phase angle $\alpha$ are the unknowns. Letting $\varphi(\tau)=\kappa \Lambda \tau+\alpha$ and substituting equation (12) into equation (1c) yields

$$
g[x(\varphi)]= \begin{cases}u_{m}-1+u_{a} \sin \varphi, & \varphi \in\left[0, \varphi_{1}^{-}\right) \quad \text { or } \quad \varphi \in\left(\varphi_{1}^{+}, 2 \pi\right], \\ 0, & \varphi \in\left[\varphi_{1}^{-}, \varphi_{2}^{-}\right] \text {or } \varphi \in\left[\varphi_{2}^{+}, \varphi_{1}^{+}\right] \\ u_{m}+1+u_{a} \sin \varphi, & \varphi \in\left(\varphi_{2}^{-}, \varphi_{2}^{+}\right),\end{cases}
$$

where $\varphi_{1}^{ \pm}=\pi+\sin ^{-1} \gamma_{ \pm}$and $\varphi_{2}^{ \pm}=2 \pi-\sin ^{-1} \gamma_{ \pm}$are transition angles that correspond to the zeros of the equation $x(\varphi)= \pm 1$, respectively, with $\gamma_{ \pm}=\left(u_{m} \pm 1\right) / u_{a}$. Equation (13) can be approximated as a harmonic expression in terms of the so-called describing functions $v_{m}$ and $v_{a}$ :

$$
g[x(\varphi)] \cong v_{m}+v_{a} \sin (\kappa \theta+\alpha) .
$$

The describing functions $v_{m}$ and $v_{a}$ are given below for the three possible contact regimes shown in Figure 2: no impact $(N I)$ where $u_{m}-u_{a}>1$; single sided impact $(S S I)$ where $\left|u_{m}-u_{a}\right| \leqslant 1$; and double sided impact $(D S I)$ where $u_{m}-u_{a}<-1$ :

$$
v_{m}=\frac{1}{2 \pi} \int_{0}^{2 \pi} g[x(\varphi)] \mathrm{d} \varphi= \begin{cases}u_{m}-1, & N I, \\ \frac{1}{2}\left(u_{m}-1\right)+A^{-} / \pi, & S S I, \\ u_{m}+\left(A^{-}-A^{+}\right) / \pi, & D S I,\end{cases}
$$




$$
\begin{gathered}
v_{a}=\frac{1}{\pi} \int_{0}^{2 \pi} g[x(\varphi)] \sin \varphi \mathrm{d} \varphi= \begin{cases}u_{a}, & N I, \\
\frac{1}{2} u_{a}+B^{-} / \pi, & S S I, \\
u_{a}+\left(B^{-}-B^{+}\right) / \pi, & D S I,\end{cases} \\
A^{ \pm}=\left(u_{m} \pm 1\right) \sin ^{-1} \gamma_{ \pm}+u_{a} \sqrt{1-\gamma_{ \pm}^{2}}, \\
B^{ \pm}=u_{a} \sin ^{-1} \gamma_{ \pm}+\left(u_{m} \pm 1\right) \sqrt{1-\gamma_{ \pm}^{2}} .
\end{gathered}
$$

Substituting equations (12) and (14) into equation (11) and enforcing harmonic balance yields the following set of non-linear algebraic equations:

$$
\begin{gathered}
v_{a}-\kappa^{2} \Lambda^{2} u_{a}+v_{m} w_{a} \cos \alpha-f_{a} \cos (\Gamma-\alpha)=0, \\
2 \zeta \kappa \Lambda u_{a}-v_{m} w_{a} \sin \alpha+f_{a} \sin (\Gamma-\alpha)=0, \\
v_{m}-f_{m}+\frac{1}{2} v_{a} w_{a} \cos \alpha=0 .
\end{gathered}
$$

The above equations may be solved for $u_{m}, u_{a}$ and $\alpha$ separately for each impact regime by substituting the appropriate describing functions from equations (14b, c). An expression for the transition frequencies separating linear $N I$ and non-linear $S S I$ solution regimes is readily obtained for the special case $f_{a}=0$ by solving for the values of $\Lambda$ and $\alpha$ which satisfy the condition $u_{m}-u_{a}=1$. The transition frequency at both sides of the $\kappa$ th superharmonic resonance is given by

$$
\Lambda_{1,2}=\frac{1}{\kappa} \sqrt{1-2 \zeta^{2} \mp \sqrt{w_{a}^{2}-4 \zeta^{2}\left(1-\zeta^{2}\right)}} .
$$

The above equation is accurate in regions of the $\kappa$ th superharmonic resonance since the steady state solution is very nearly harmonic with frequency $\kappa \Lambda$ in these regimes regardless of the harmonic content of $\psi$; this is demonstrated later in Section 3.5.

For a special case of a lightly damped system in which $\zeta \ll 1$, equation (16) reduces to $\Lambda_{1,2} \simeq \sqrt{1 \mp w_{a}} / \kappa$. Furthermore, since the discriminant $w_{a}^{2}-4 \zeta^{2}\left(1-\zeta^{2}\right)>0$ in order for the transition frequencies to be real, all solutions must be in the NI regime so long as $w_{a}<2 \zeta$. Hence, the existence of separations is dictated entirely by $w_{a}$ and $\zeta$ for lightly damped systems $(\zeta<0 \cdot 1)$ and is completely independent of preload $f_{m}$.

\subsection{GENERAL HBM SOLUTION IN THE $N I$ REGIME}

A general solution for the linear $N I$ case is obtained by substituting $v_{1}=1+u_{1}$ and $v_{i}=u_{i}$, $i \in[1,2 R+1]$ into equation (3). The conditions $\mathbf{S}=\mathbf{0}$ is satisfied by solving the linear equation $\mathbf{A u}=\mathbf{b}$, where the elements of square matrix $\mathbf{A}$ and column vector $\mathbf{b}$, both of dimension $(2 R+1)$, are given respectively by

$$
\begin{gathered}
a_{11}=2, \\
a_{1,2 i}=a_{2 i, 1}=w_{2 i}, \quad i \in[1, R], \\
a_{1,2 i+1}=a_{2 i+1,1}=w_{2 i+1}, \quad i \in[1, R],
\end{gathered}
$$




$$
\begin{aligned}
& a_{2 i, 2 i}=1-(i \Lambda)^{2}+\frac{1}{2} w_{4 i}, \quad i \in[1, R], \\
& a_{2 i, 2 i+1}=2 \zeta(i \Lambda)+\frac{1}{2} w_{4 i+1}, \quad i \in[1, R], \\
& a_{2 i+1,2 i}=-2 \zeta(i \Lambda)+\frac{1}{2} w_{4 i+1}, \quad i \in[1, R], \\
& a_{2 i+1,2 i+1}=1-(i \Lambda)^{2}-\frac{1}{2} w_{4 i}, \quad i \in[1, R], \\
& a_{2 i, 2 j}=a_{2 j, 2 i}=\frac{1}{2}\left[w_{2(j-i)}+w_{2(j+i)}\right], \quad i \in[1, R], \quad j \in[1,(i-1)], \\
& a_{2 i, 2 j+1}=a_{2 j+1,2 i}=\frac{1}{2}\left[-w_{2(j-i)+1}+w_{2(j+i)+1}\right], \quad i \in[1, R], \quad j \in[1,(i-1)], \\
& a_{2 i+1,2 j}=a_{2 j, 2 i+1}=\frac{1}{2}\left[w_{2(j-i)+1}+w_{2(j+i)+1}\right], \quad i \in[1, R], \quad j \in[1,(i-1)], \\
& a_{2 i+1,2 j+1}=a_{2 j+1,2 i+1}=\frac{1}{2}\left[w_{2(j-i)}-w_{2(j+i)}\right], \quad i \in[1, R], \quad j \in[1,(i-1)], \\
& \mathbf{b}=\left\{\begin{array}{llllllllllll}
2 f_{1}+2 & w_{2} & w_{3} & w_{4} & w_{5} & \ldots & w_{2 K} & w_{2 K+1} & 0 & 0 & 0 & \ldots
\end{array}\right\}^{\mathrm{T}} .
\end{aligned}
$$

Solving the above system of equations directly requires considerably less computational effort than the Newton-Raphson procedure defined by equation (6). This leads to an efficient general algorithm to solve the governing equation (1). In NI regimes bounded approximately by the transition frequencies given by equation (16), the above set of equations is solved directly for $\mathbf{u}$ by using a Gaussian elimination procedure [23], whereas equation (6) is employed to determine $\mathbf{u}$ in $S S I$ and $D S I$ regimes.

\section{EXPERIMENTAL VALIDATION}

In order to validate equation (1) and demonstrate several non-linear phenomena exhibited by the mechanical oscillator of Figure 1, a unique gear dynamics test rig was designed and constructed. Specialized instrumentation and data acquisition and signal processing software were developed to measure in real time the vibratory displacement of a gear pair operating under conditions typical of an industrial rotating machine. Parameters required by equation (1) were determined experimentally and analytical solutions obtained by HBM and numerical integration are compared with experimental steady state measurements.

\subsection{APPLICATION EXAMPLE: A GEAR PAIR}

As a practical application of the proposed governing equation (1), consider a purely torsional two-degree-of-freedom (DOF) model of a gear pair supported by rigid mounts as shown in Figure 3. The rotational angle of each gear $\theta^{i}(t)=\Omega^{i} t+\theta_{a}^{i}(t)$ is written as the sum of a nominal rotation angle $\Omega^{i} t$ and a vibratory component $\theta_{a}^{i}(t)$ which arises due to the gear meshing action. Here $t$ represents real time, and $\Omega^{i}$ is the mean rotational velocity of gear $i$. The $\Omega^{i}$ and $\Omega^{j}$ are related kinematically by $\rho^{i} \Omega^{i}=\rho^{j} \Omega^{j}$, where $\rho^{i}$ is the base radius of gear $i$. Since the system is semi-definite, the dynamic model may be reduced to a single DOF in terms of the so-called dynamic transmission error co-ordinate $\xi(t)=\rho^{i} \theta_{a}^{i}(t)+\rho^{j} \theta_{a}^{j}(t)$, which represents relative displacement across the gear mesh interface. Here, subscripts $i$ and $j$ denote gears $i$ and $j$ respectively. The corresponding equation of motion is given by

$$
\begin{gathered}
m_{e q} \xi^{\prime \prime}+c_{e q} \xi^{\prime}+k\left[\theta^{*}(t)\right] \hat{g}\left\{\xi(t)-\epsilon\left[\theta^{*}(t)\right]\right\}=F, \\
\hat{g}\left\{\xi(t)-\epsilon\left[\theta^{*}(t)\right]\right\}= \begin{cases}\xi(t)-\epsilon\left[\theta^{*}(t)\right]-b, & \xi(t)-\epsilon\left[\theta^{*}(t)\right]>b, \\
0, & \left|\xi(t)-\epsilon\left[\theta^{*}(t)\right]\right| \leqslant b, \\
\xi(t)-\epsilon\left[\theta^{*}(t)\right]+b, & \zeta(t)-\epsilon\left[\theta^{*}(t)\right]<-b .\end{cases}
\end{gathered}
$$




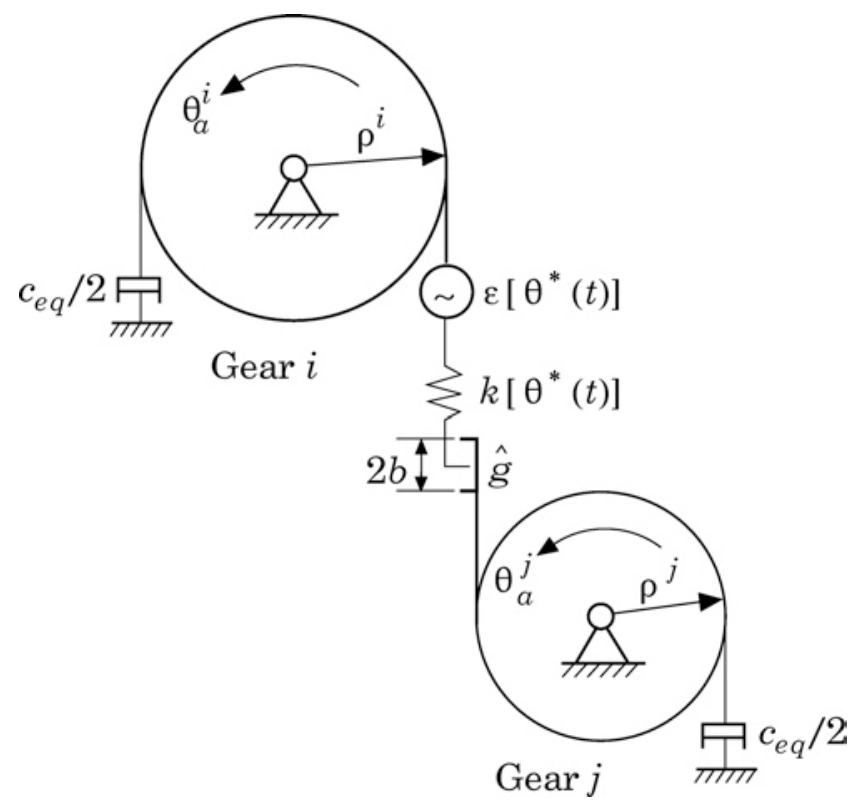

Figure 3. An application example: a single-DOF torsional model of a gear pair.

Here, (') denotes differentiation with respect to $t, m_{e q}=I^{i} I^{i} /\left[I^{i}\left(\rho^{i}\right)^{2}+I^{i}\left(\rho^{i}\right)^{2}\right]$ is the system equivalent mass determined from the polar mass moment of inertia $I^{i}$ of each gear, $c_{e q}$ is an equivalent viscous damping coefficient which describes frictional losses of the system, $F$ is the mean load carried by the gear teeth and $2 b$ is the total backlash. The instantaneous mesh stiffness of the gear pair $k\left[\theta^{*}(t)\right]$ varies periodically according to the number of teeth in contact for a given system rotation angle $\theta^{*}(t)=\Omega^{*} t+\beta \xi(t)$. The term $\epsilon\left[\theta^{*}(t)\right]$ describes manufacturing errors associated with mating gear teeth and must also be periodic in $\theta^{*}$. Thus, $\epsilon$ gives rise to external forcing which may be neglected for precision gear pairs with no intentional tooth flank modifications. The system rotational angle is written in terms of the system velocity $\Omega^{*}=\Omega^{i} / n^{i}$ and modulation depth $\beta=n^{i} / \rho^{i}$, where $n^{i}=N^{i} / G C F\left(N^{i}, N^{j}\right)$ is an integer and $G C F\left(N^{i}, N^{j}\right)$ is the greatest common factor of gear tooth numbers $N^{i}$ and $N^{j}$. Hence the fundamental frequency $\Omega^{*}$ of $k$ and $\epsilon$ is $n^{i} N^{i}$ times lower than the so-called gear meshing or tooth engagement frequency $\Omega=n^{i} N^{i} \Omega^{i}$. However, if tooth-to-tooth variations of each gear are kept small by precision manufacturing, then both $k$ and $\epsilon$ may be assumed to be periodic at the meshing frequency $\Omega$.

Let $\tau=\omega_{n} t$ be a dimensionless time parameter, where $\omega_{n}=\sqrt{k_{m} / m_{e q}}$ is the undamped natural frequency of the corresponding linear time-variant system and $k_{m}$ is the mean value of $k[\theta(\tau)]$. Accordingly, the equation of motion (21) may be reduced to the governing equation (1) for the case $\epsilon\left[\theta^{*}(t)\right]=0$ by defining $x(\tau)=\xi(\tau) / b, \zeta=c_{e q} / 2 \sqrt{m_{e q} k_{m}}$ and $\psi[\theta(\tau)]=k[\theta(\tau)] / k_{m}$. The dimensionless forcing frequency is given by $\Lambda=\Omega / \omega_{n}$ and $f[\theta(\tau)]=f_{1}=F / k_{m} b$ in accordance with the mean and alternating convention defined earlier.

The above single-DOF model is valid for precision gear pairs provided that the equivalent translational stiffness of shafting, bearings and supporting structures are sufficiently large relative to the equivalent mesh stiffness [4]. In cases in which the amplitude of $\xi(t)$ is relatively small (less than $10 \mu \mathrm{m}$ ), angle modulation effects are negligible and the assumption $\beta=0$ is valid [1].

\subsection{EXPERIMENTAL GEAR DYNAMICS TEST RIG}

The four-square gear test rig shown in Figure 4 was designed precisely to measure $\xi(t)$ for a gear pair operating under a range of speed and load conditions. The lubrication bonnet and several guards have been removed for clarity in this photograph. Several unique features are required in order to ensure that the behavior of the test rig is indeed described by equation 


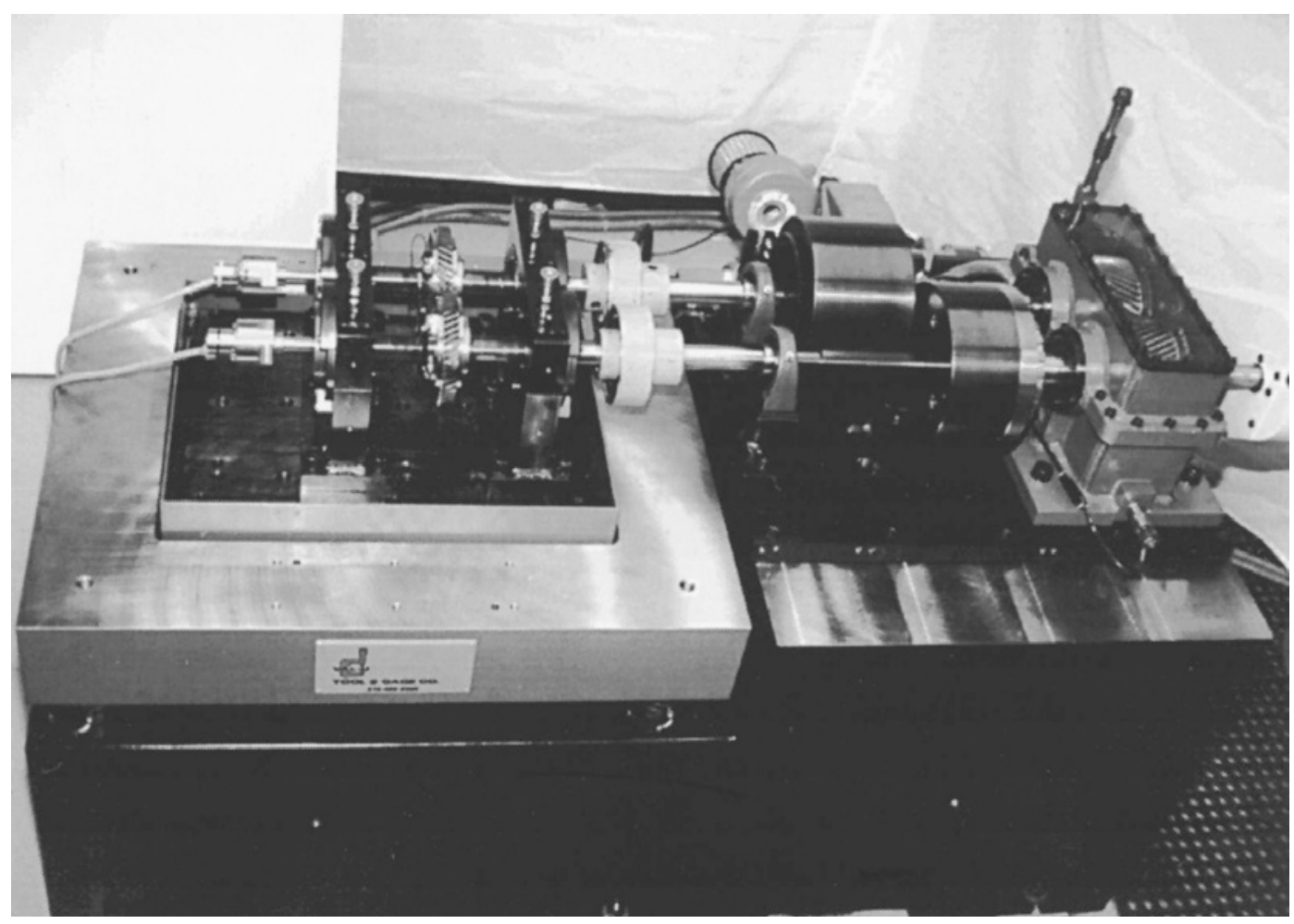

Figure 4. The experimental test rig.

(1). These features are labelled in the schematic diagram shown in Figure 5. A precision test gear pair (i) is straddle mounted between oversized ultra-precision spherical roller bearings (ii) which are themselves supported by rigid bearing pedestals (iii). The span of the test gear shafts (iv) is kept to an absolute minimum and the shafting diameter is sufficiently large to ensure that any translational or shaft bending modes are well above the primary torsional resonance of the test gear pair so that the SDOF model is valid. The test gear pair is dynamically isolated from the slave gear box (v) by compliant elastomer couplings (vi) and flywheels (vii). Precision helical slave gears (viii) are machined with a different number of teeth from the test gears so that the meshing frequencies are not commensurate. Furthermore, the slave gear tooth flanks have specially ground topological modifications to ensure minimal excitation over a range of operating loads. A power recirculation arrangement is employed to ensure precise torque regulation and efficient operation. System torque is manually adjusted by loosening a split hub coupling (ix) and applying a static torque using a locking pin (x) and a removable torque arm with dead weights that are not shown. Once system torque is applied, the split hub is tightened and the torque arm removed. Power is supplied by a DC motor (xi) equipped with electronic speed control. In order to keep damping to a minimum, the system is lubricated with low viscosity automatic transmission fluid under controlled elevated temperatures. Lubrication is applied to the gear mesh exit and all four bearings at controlled flow rates to ensure consistent film thickness and hence constant damping ratio. Experimental investigations indicated that the system damping is dictated primarily by the bearings under the above lubrication conditions; thus employing a linear viscous damping term in equation (19a) is valid even when tooth separations take place.

The test gears are of a standard 50 tooth, $3.0 \mathrm{~mm}$ module, $20^{\circ}$ pressure angle unity ratio design such that $\rho=70.477 \mathrm{~mm}$ and were ultra-precision ground with no intentional flank modifications such that $\epsilon=0$ and $f_{a}=0$. The spectral content of $k_{a}$ is dictated primarily by the so-called involute contact ratio which may be varied over a suitable range by selecting mating gears of the same nominal design but having different major diameters. The mean stiffness $k_{m}$ depends mostly on the gear face width and involute contact ratio. Accordingly, 

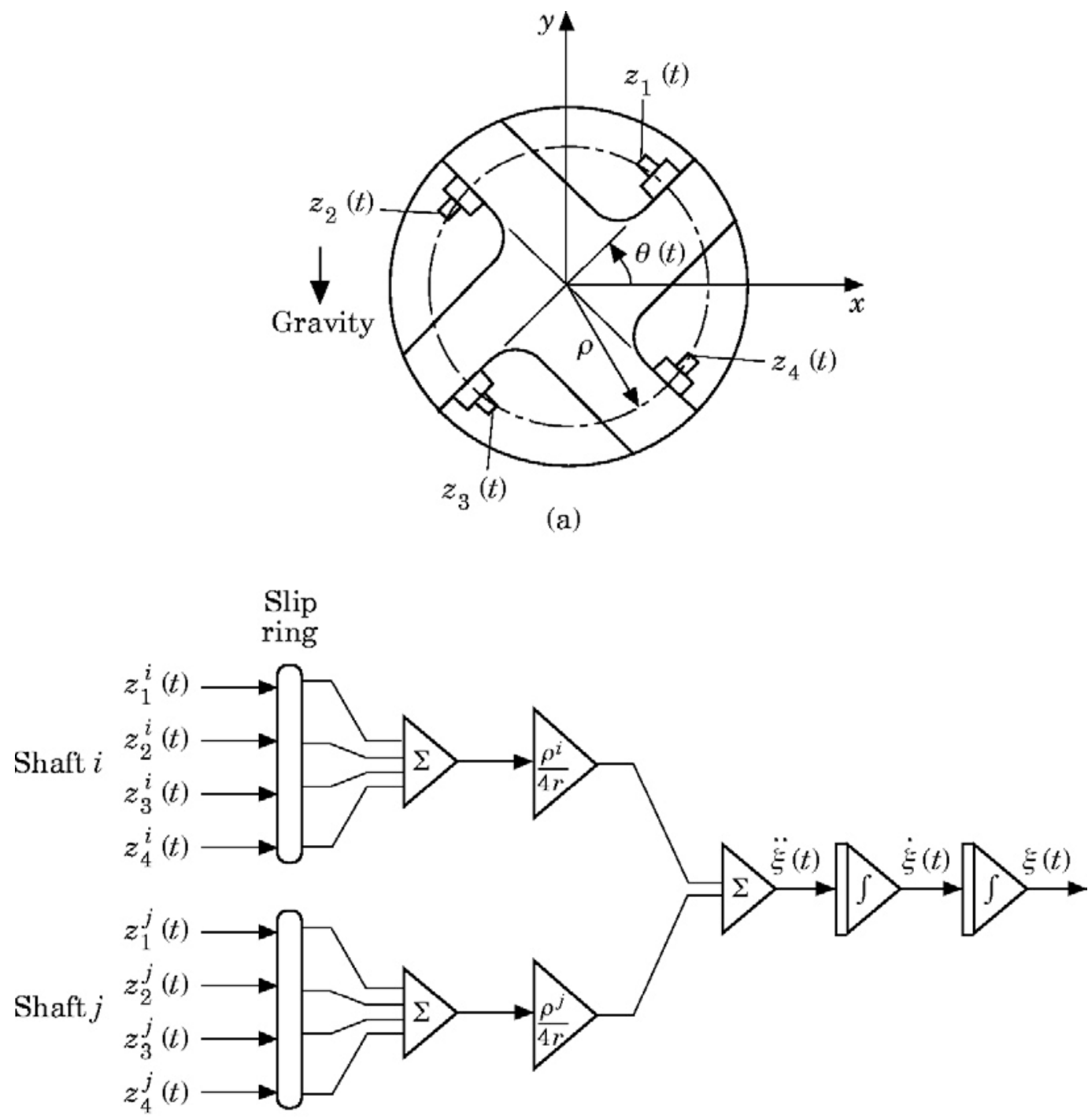

(b)

Figure 6. (a) The arrangement of linear accelerometers on a gear wheel and (b) the block diagram of the analog circuitry used to compute $\xi(t)$ from measured accelerometer signals $z_{i}(t)$.

which may occur. Although two diametrically opposed accelerometers are sufficient, four are used in order to achieve higher measurement accuracy by averaging random errors. The resulting angular acceleration of each gear is then added in the time domain to obtain $\xi^{\prime \prime}(t)$ and subsequently integrated twice to yield $\xi^{\prime}(t)$ and $\xi(t)$, respectively, by using the analog circuitry shown in the block diagram of Figure 6(b). This method allows direct measurement of $\xi(t)$ and its time derivatives to an extremely high accuracy of less than $0.001 \mu \mathrm{m}$ at $1000 \mathrm{~Hz}$. The measurement accuracy is highly dependent upon the excitation frequency, since displacement is directly proportional to the measured acceleration amplitude by the square of the excitation frequency.

\subsection{DATA ACQUISITION AND SIGNAL PROCESSING}

Analog signals representing $\xi(t)$ and $\xi^{\prime}(t)$ are captured and analyzed in real time by using a high speed multiple channel signal analysis workstation. Synchronous time averaging is employed by controlling the analyzer sampling rate from a clock pulse obtained from a high precision 18000 line optical encoder (xiv) mounted on the test gear shaft. This not only results in a high signal to noise ratio but, more importantly, allows all analyses to be easily conducted with respect to dimensionless time $\tau$ once $\omega_{n}$ is known from an experimental modal analysis. Averaging also diminishes the influence of any slight differences among individual teeth that exist due to unavoidable manufacturing errors; the effects of which were otherwise neglected by the periodicity assumptions in Section 3.1. All Fourier transforms 
are performed in the so-called orders domain rather than the frequency domain. The measured $\xi(t)$ and $\xi^{\prime}(t)$ are normalized to obtain $x(\tau)$ and $x^{\prime}(\tau)$ respectively. The $r$ th harmonic amplitude $A_{r}$ of $x(\tau)$ is determined by

$$
A_{r}=\sqrt{\sum_{s^{i}=N^{i}-B W / 2}^{N_{i}+B W / 2} \tilde{X}\left(r s^{i}\right) \tilde{X}^{*}\left(r s^{i}\right)}
$$

where $\tilde{X}\left(s^{i}\right)$ is the complex valued discrete Fourier coefficient of $x(\tau)$ corresponding to shaft order index $s^{i}, N^{i}$ is the number of teeth on gear $i, B W$ is the analysis bandwidth in shaft orders and $(*)$ denotes complex conjugate. A typical value of $B W=4$ was chosen in order to account for sidebands and leakage and bin errors inherent to experimental spectrum analysis. An equivalent root-mean-square (r.m.s.) amplitude $A_{r m s}$ of the alternating component of $x(\tau)$ is given by

$$
A_{r m s}=\sqrt{\sum_{r=1}^{R} A_{r}^{2}},
$$

where the maximum number of harmonic terms $R$ available to approximate $A_{\text {rms }}$ depends on the operating mesh frequency range $200 \leqslant \Omega \leqslant 3400 \mathrm{~Hz}$ and the upper frequency limitation of the angular accelerometer $\Omega \leqslant 5000 \mathrm{~Hz}$. Although the alternating component of $x(\tau)$ can be measured very accurately, the mean value of $x(\tau)$ cannot be obtained from accelerometer measurements.

\subsection{SOLUTION VALIDATION}

Experimental values of $A_{\text {rms }}$ are compared with equivalent r.m.s. amplitudes obtained from a three-term harmonic balance solution in Figure 7 over the frequency range $0 \cdot 2 \leqslant \Lambda \leqslant 1 \cdot 1$, which includes the fundamental and first two superharmonic resonances. Stiffness values $w_{i}$ were determined experimentally for a normalized preload of $f_{1}=1 \cdot 0$, given $\omega_{n}=19500 \mathrm{rad} / \mathrm{s}$ and $b=0.015 \mathrm{~mm}$. The value of $\zeta$ was estimated to be 0.01 from experimental data. Also shown in Figure 7 are corresponding solutions obtained by direct

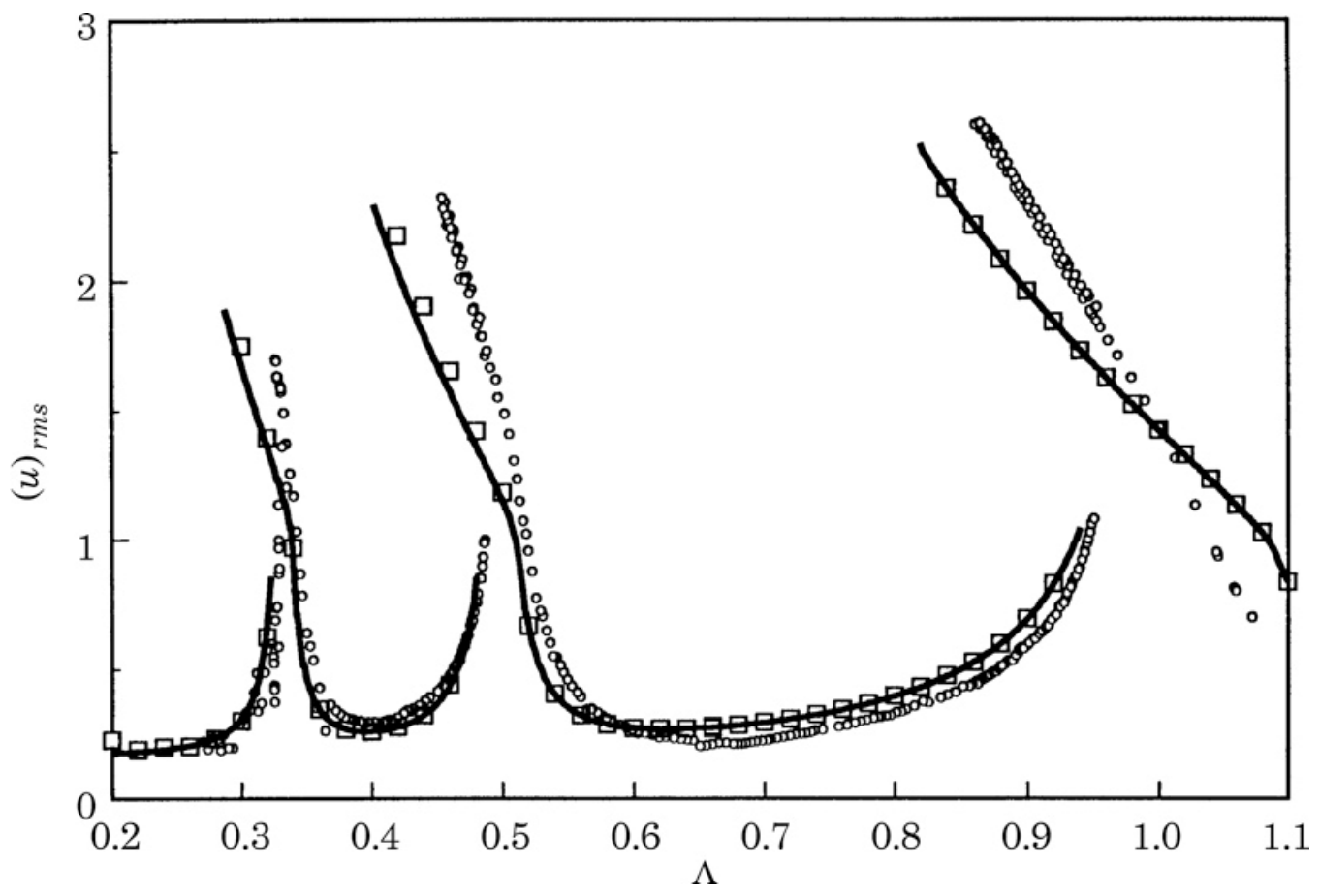

Figure 7. A comparison of HBM solutions $(-)$ with numerical integration $(\square)$ and experimental $(\bigcirc)$ results. $R=3, K=3$, with $w_{2}=0 \cdot 15, w_{4}=0 \cdot 075, w_{6}=0.06$ and all other $w_{i}=0, f_{1}=1 \cdot 0$ with all other $f_{i}=0$, and $\zeta=0 \cdot 01$. 

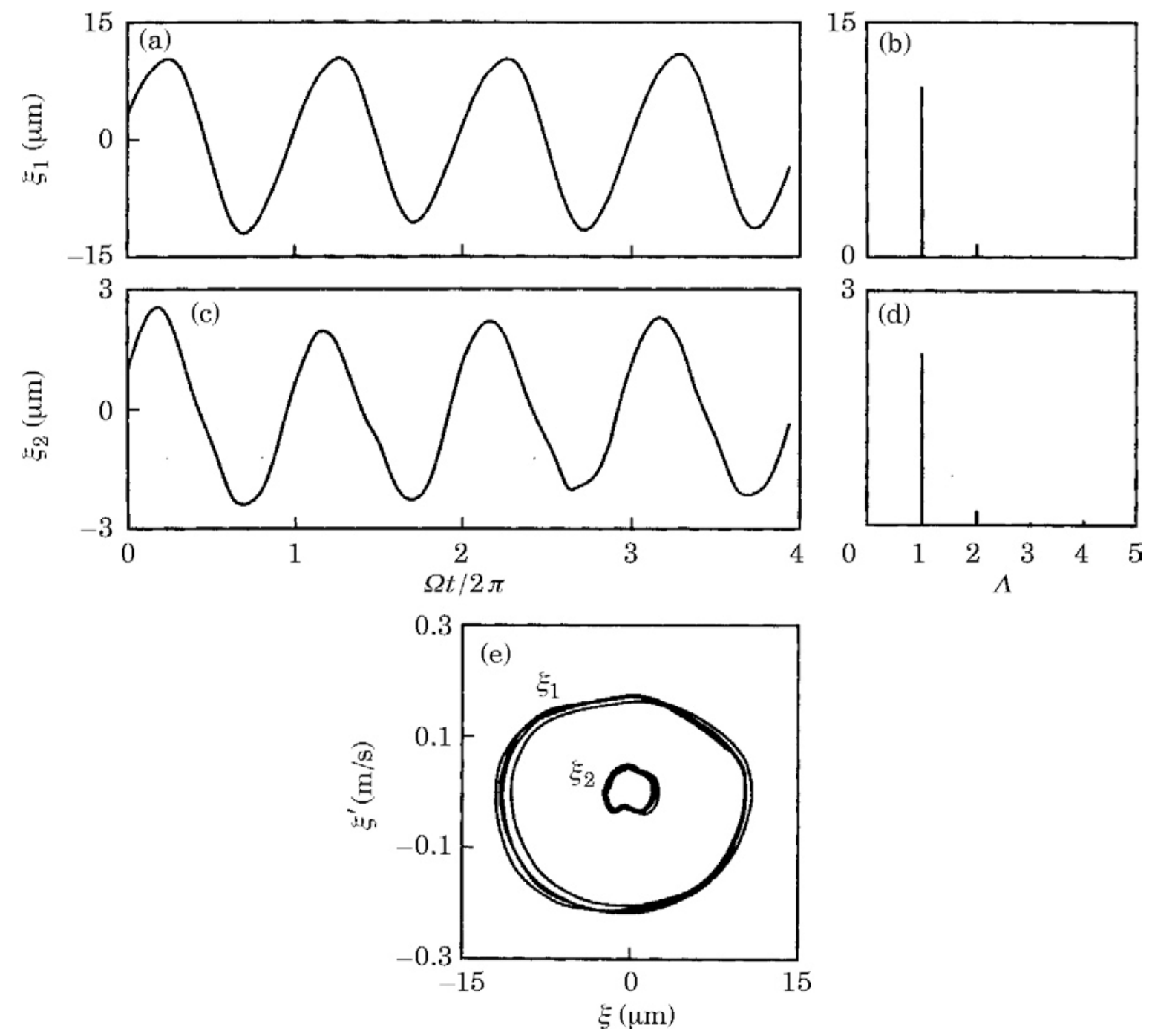

Figure 8. Measured coexisting steady state solutions $\xi_{1}(t)$ and $\xi_{2}(t)$ at $\Lambda=0 \cdot 9$ : (a) $\xi_{1}(t)$ versus $\Omega t / 2 \pi$; (b) FFT of $\xi_{1}(t)$; (c) $\xi_{2}(t)$ versus $\Omega t / 2 \pi$; (d) the FFT of $\xi_{2}(t)$; and (e) phase portraits $\xi_{1}^{\prime}(t)$ versus $\xi_{1}(t)$ and $\xi_{2}^{\prime}(t)$ versus $\xi_{2}(t)$. The system parameters are the same as in Figure 7.

time domain numerical integration of equation (1) by using a fifth-sixth order variable step size Runge-Kutta algorithm that are in excellent agreement with the HBM solution. Overall, the analytical solution matches quite well with the experimental data which exhibited only $N I$ and SSI responses for the particular physical configuration tested. The response of the physical system was extremely well behaved and repeatable. In regions of multiple solutions, the lower branch was obtained by slowly increasing the system speed until jump-up occurred. Similarly, the upper branch was obtained by slowly decreasing system speed until jump-down occurred. The frequencies at which these transitions occurred were very distinct and could be detected audibly by a 10-40 dB change in sound level. Differences between analytical and experimental results in the upper solution branches are attributed to inaccuracies in the simplified mesh interface model as well as slight manufacturing variances that caused $\epsilon$ and $f_{a}$ to be other than zero under actual operating conditions.

The measured time domain response of the system within double solution regimes in the vicinity of the fundamental $(\kappa=1)$ and first superharmonic $(\kappa=2)$ resonance is shown in Figures 8 and 9 , given $\Lambda=0 \cdot 9$ and $\Lambda=0 \cdot 45$, respectively. The measurements are presented in dimensional form in these two figures in order to demonstrate how small the amplitude of $\xi$ is in practice. In both cases, the measured response is nearly harmonic with frequency $\kappa \Lambda$. The harmonic content of the analytical solution presented in Figure 7 is examined by plotting the forced response curves $A_{r m s}, A_{1}, A_{2}$ and $A_{3}$ versus $\Lambda$ for $N I$ and $S S I$ solutions only, as shown in Figure 10. Clearly, the steady state forced response in the vicinity of the $\kappa$ th resonance is nearly harmonic so that $A_{r m s} \cong A_{\kappa}$. The amplitude $A_{\kappa}$ is dictated almost 

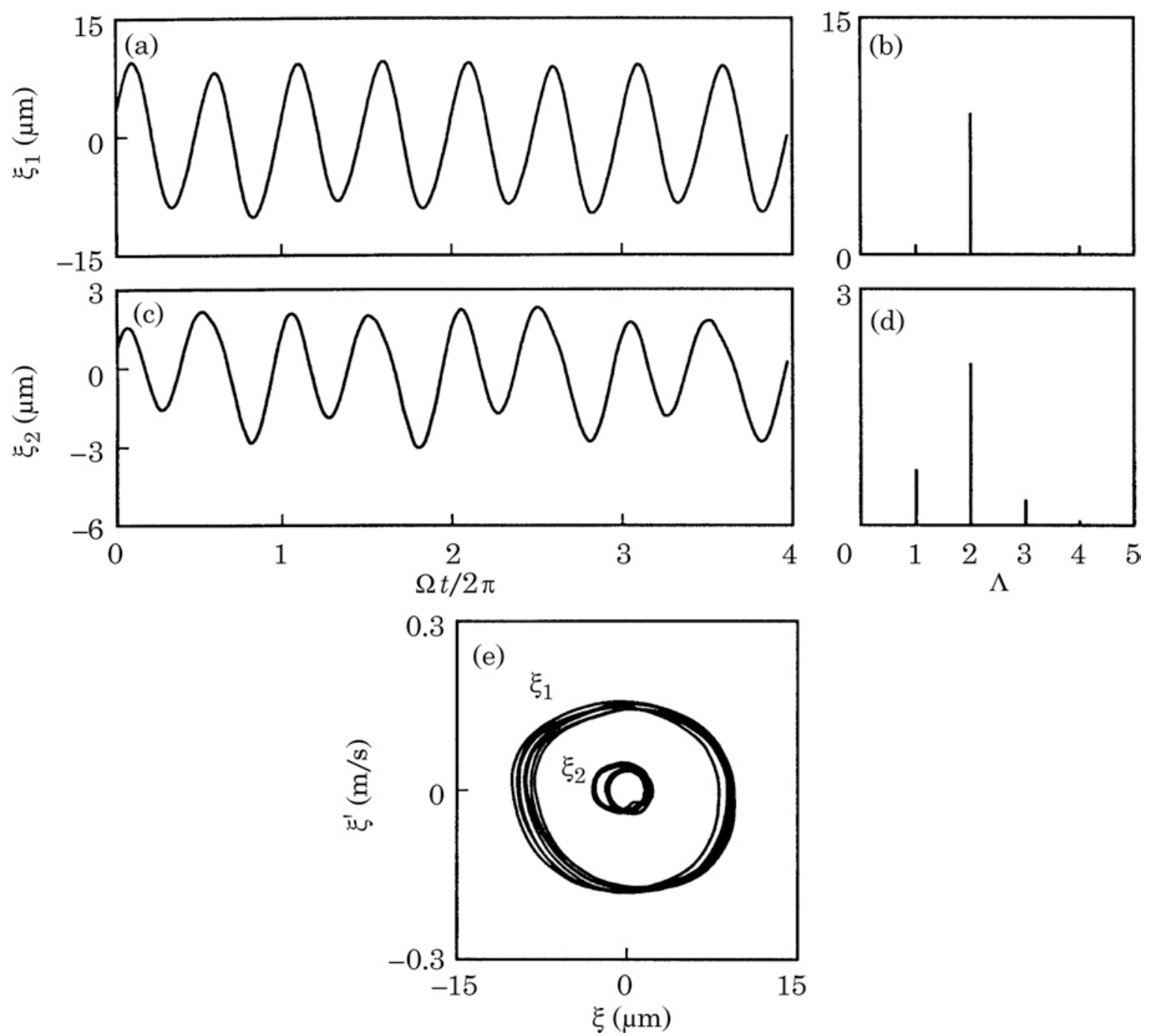

Figure 9. Measured coexisting steady state solutions $\xi_{1}(t)$ and $\xi_{2}(t)$ at $\Lambda=0 \cdot 45$ : (a) $\xi_{1}(t)$ versus $\Omega t / 2 \pi$; (b) the FFT of $\xi_{1}(t)$; (c) $\xi_{2}(t)$ versus $\Omega t / 2 \pi$; (d) the FFT of $\xi_{2}(t)$; (e) phase portraits $\xi_{1}^{\prime}(t)$ versus $\xi_{1}(t)$ and $\xi_{2}^{\prime}(t)$ versus $\xi_{2}(t)$. The system parameters are the same as in Figure 7.

entirely by the $\kappa$ th harmonic stiffness amplitudes $w_{2 \kappa}$ and $w_{2 \kappa+1}$ and is influenced only slightly by stiffness harmonics other than those of frequency $\kappa \Lambda$. Hence, for practical purposes, the system response in regions near the fundamental and superharmonic resonances may be described by a single-term harmonic balance solution at frequency $\kappa \Lambda$. However, additional harmonic terms must be included in the corresponding NI solution in off-resonance regimes. Also shown in Figure 10 are the corresponding r.m.s. and harmonic amplitudes obtained from an LTV analysis by neglecting backlash completely. Note that the LTV solution becomes unstable in the vicinity of the fundamental $(\kappa=1)$ resonance for the lightly damped case considered here. This occurs because of an unstable parametric resonance attributed to the second harmonic stiffness terms $w_{4}$ and $w_{5}$ that coincides with the otherwise stable $\kappa=1$ resonance [1]. Hence, the clearance non-linearity has a stabilizing effect on the forced response which is in exact agreement with the observed behavior of the physical system.

\section{PARAMETRIC STUDIES: HARMONIC SOLUTION}

The system response has been shown to be nearly harmonic in resonance regimes where the presence of the clearance type non-linearity dictates the system behavior. Accordingly, the harmonic solution of Section 2.2 is employed to study the effects of $w_{a}, \zeta$ and $f_{m}$ on the steady state forced response of a lightly damped system, $\zeta \leqslant 0 \cdot 1$, with an emphasis on the fundamental and superharmonic resonance regimes. 


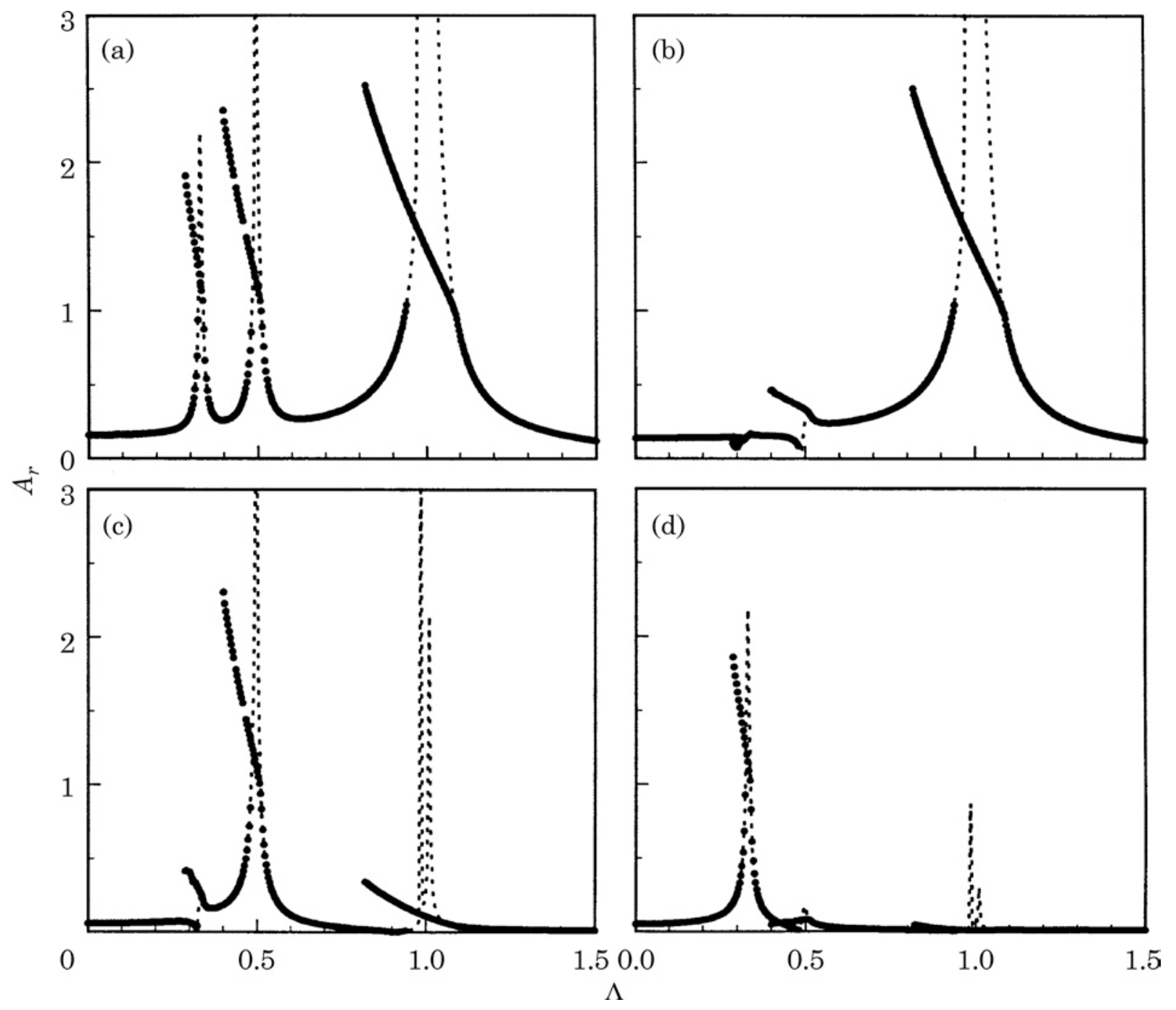

Figure 10. A comparison of the harmonic content of multiple term HBM solutions $(\bullet)$ with the corresponding LTV system results (-.-.-); (a) $A_{r m s}$ versus $\Lambda$; (b) $A_{1}$ versus $\Lambda$; (c) $A_{2}$ versus $\Lambda$; (d) $A_{3}$ versus $\Lambda$. The system parameters are the same as in Figure 7.

\subsection{EFFECT OF STIFFNESS VARIATION}

The effect of $w_{a}$ on the mean $u_{m}$ and alternating $u_{a}$ components of $x(\tau)$ is shown in Figure 11 for a given preload $f_{m}=0.5$ and damping ratio $\zeta=0 \cdot 05$. Both stable and unstable $S S I$ and DSI solutions are shown along with the linear NI portion of the steady state forced response over the frequency range $0.5 \leqslant \kappa \Lambda \leqslant 1 \cdot 5$. For $w_{a}<2 \zeta$, the system behaves in a linear fashion and no jumps or unstable solutions occur. As $w_{a}$ is increased beyond $2 \zeta$, two stable solutions coexist. The shape of the forced response curve for $w_{a}<0.25$ is similar to that of a forced Duffing's equation of the softening type. Both jump-up and jump-down frequencies and the amount of frequency overlap between the upper and lower stable solutions are highly dependent upon $w_{a}$. The lower jump-up frequency is nearly equal to the transition frequency $\Lambda_{1}$ given by equation (16) and this corresponds to a fold bifurcation. While the upper transition frequency $\Lambda_{2}$ predicted by equation (16) is evident from a corresponding inflection in the forced response curve, the actual jump-down frequency occurs at a much lower frequency. This behavior is consistent with the experimental forced response curves shown in Figure 12, which were obtained by overlaying several measured response curves $A_{\kappa}$ versus $\kappa \Lambda$, with each harmonic having a distinct value of $w_{a}$ for a given gear involute contact ratio. As $w_{a}$ is increased further such that $u_{m}-u_{a}<-1$, a $D S I$ solution is revealed which folds back to the right as shown in Figure 11 for $w_{a}>0 \cdot 25$. Transitions from NI to $S S I$ are readily detected by the deviation of $u_{m}$ from its otherwise constant value in NI regimes, as shown in Figure 11(b). 

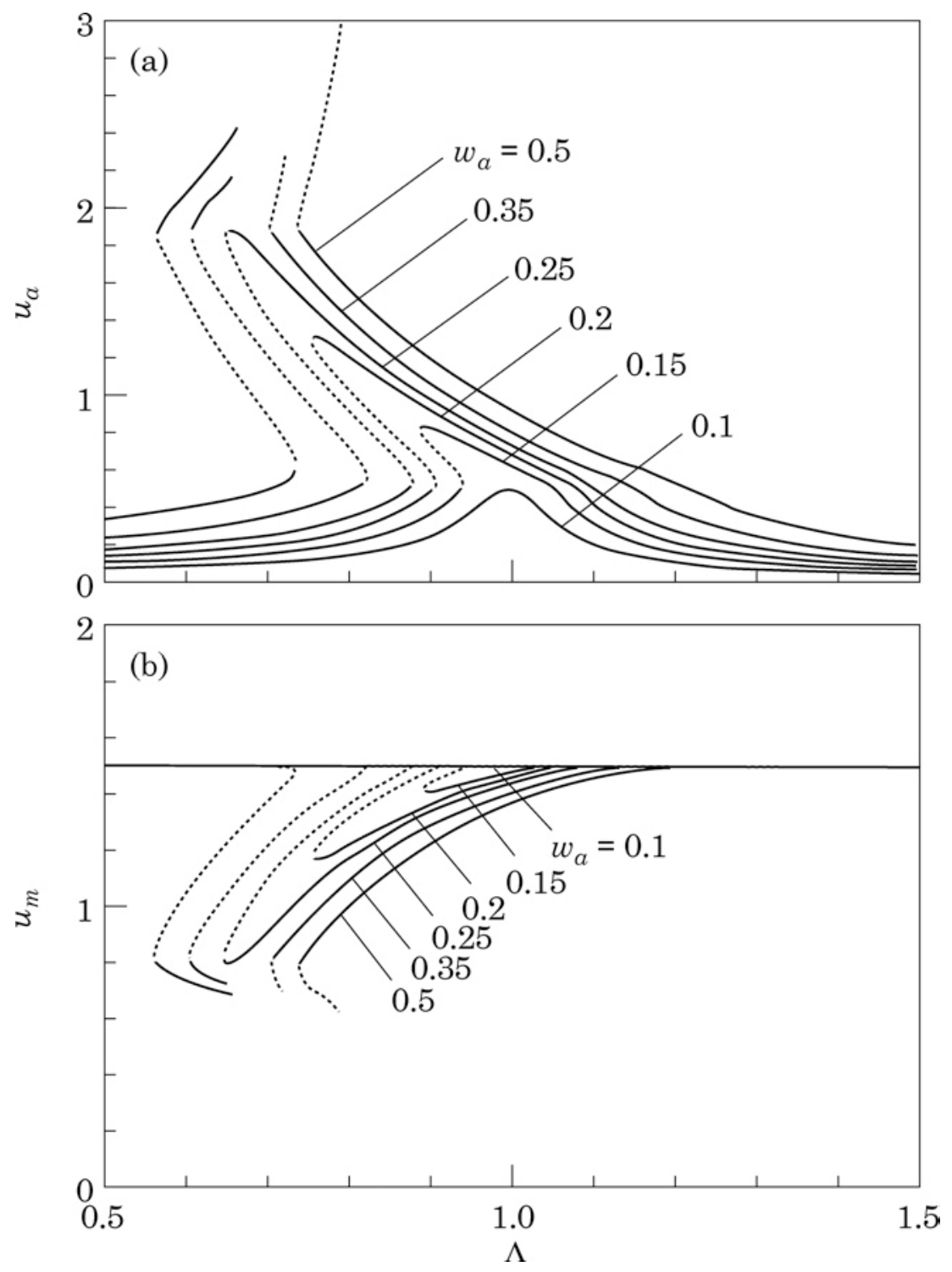

Figure 11. The effect of stiffness variation $w_{a}$ on the (a) alternating $u_{a}$ and (b) mean $u_{m}$ components of the steady state forced response for $w_{a} \in[0 \cdot 1,0 \cdot 5], f_{m}=0 \cdot 5$, and $\zeta=0 \cdot 05$.

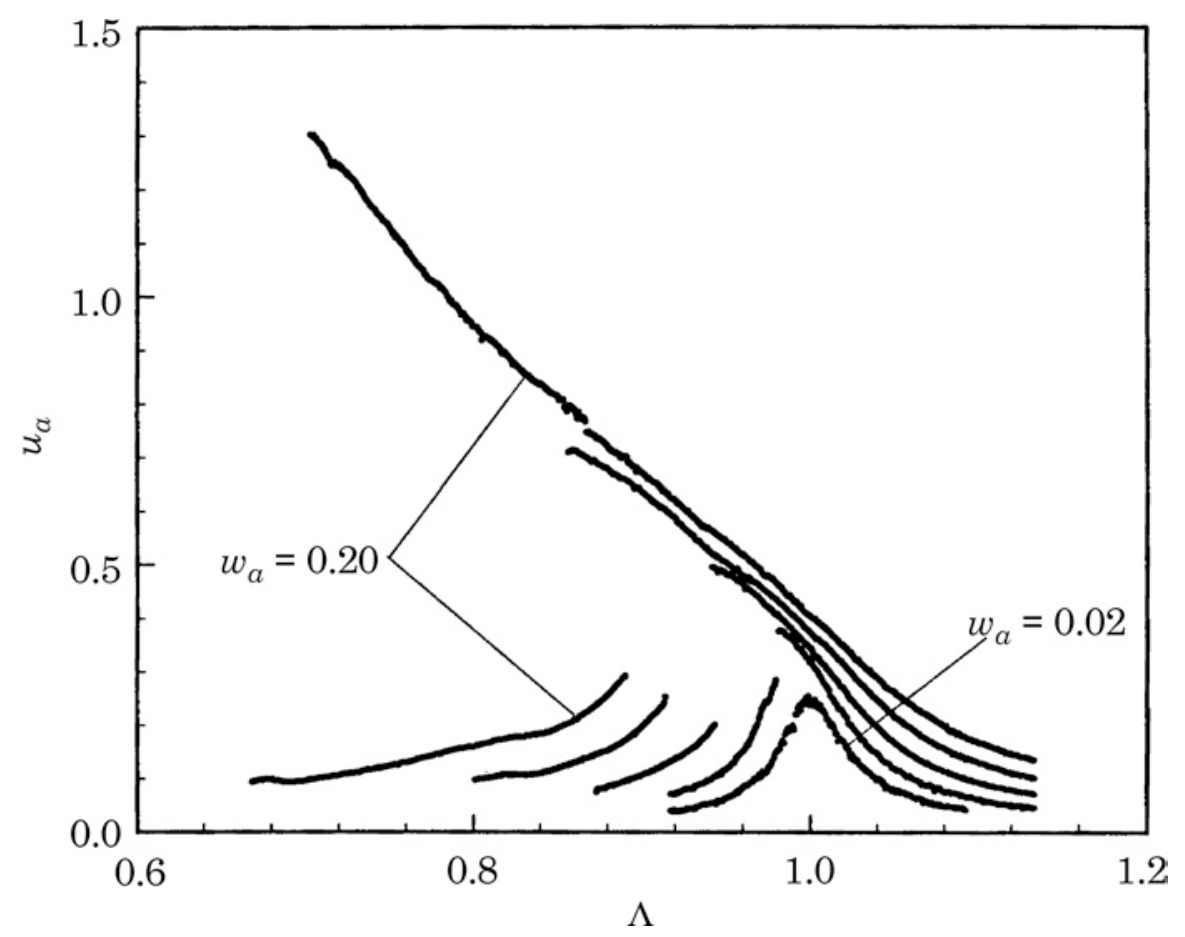

Figure 12. The effect of stiffness variation $w_{a}$ on the measured alternating component $u_{a}$ of the steady state forced response for $w_{a} \simeq 0 \cdot 20,0 \cdot 15,0 \cdot 10,0 \cdot 05$ and $0 \cdot 02, \zeta \simeq 0 \cdot 01$ and $f_{m}=0 \cdot 5$. 


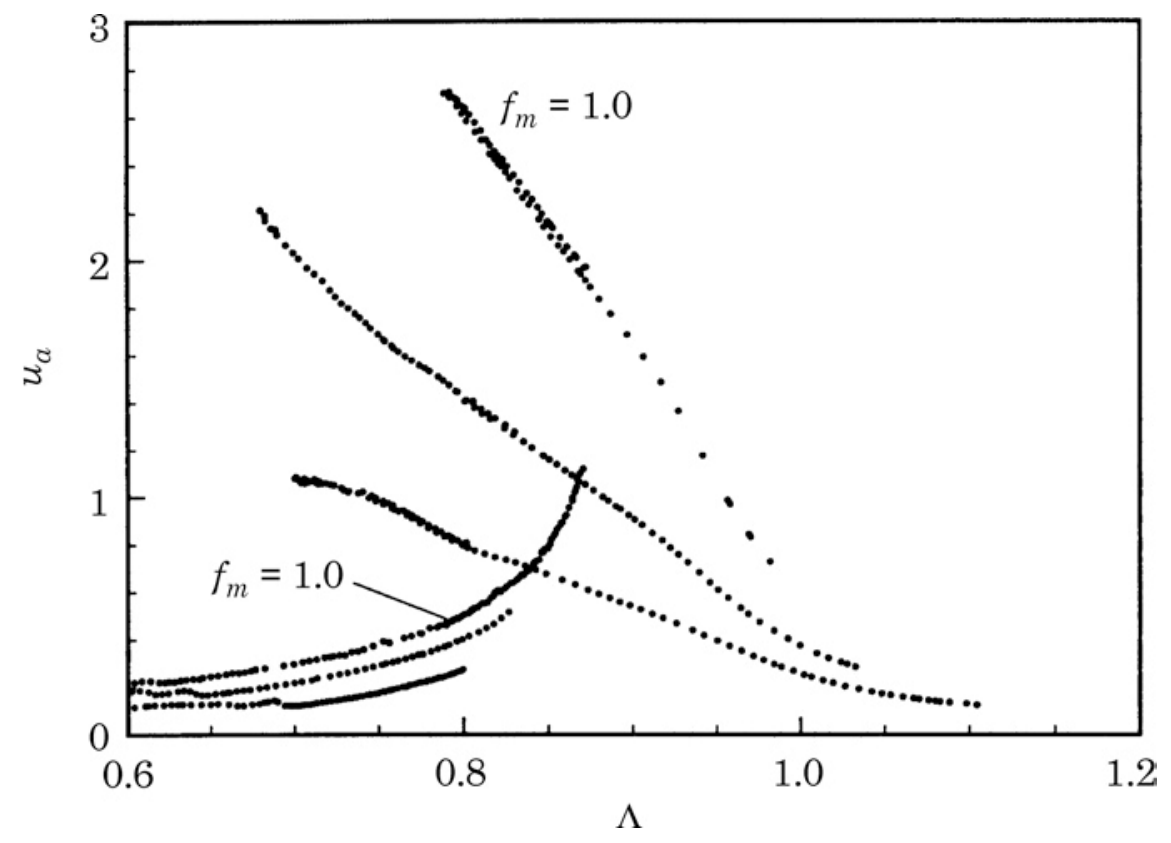

Figure 15. The effect of the preload $f_{m}$ on the measured alternating component $u_{a}$ of the steady state forced response for $f_{m} \simeq 1 \cdot 0,0 \cdot 5$ and $0 \cdot 25, w_{a} \simeq 0 \cdot 20$ and $\zeta \simeq 0 \cdot 01$.

harmonic solution is obtained which yields mathematical conditions for the occurrence of non-linear behavior in the system response.

Well behaved coexisting stable response solutions have been clearly demonstrated using a relatively common mechanical system that has significant relevance to automotive, aerospace, marine and industrial power transmission and gearing applications. The gear pair system used as the engineering application is inherently non-linear and exhibits a range of complex behavior beyond that reported in this publication. For instance, subharmonic resonances, as well as aperiodic motions and chaotic behavior, have been observed and reported in another recent paper [25].

\section{ACKNOWLEDGMENTS}

The authors wish to thank the Powertrain Division of General Motors Corporation for supporting this research effort. Mr S. Moorman and Mr T. Vogel are acknowledged for their assistance in developing signal processing software and performing experimental analyses, respectively.

\section{REFERENCES}

1. G. W. Blankenship and R. Singh 1995 Journal of Sound and Vibration 179, 13-36. Analytical solutions for modulation sidebands associated with a class of mechanical oscillators.

2. J. A. Richards 1983 Analysis of Periodically Time-varying Systems. New York: Springer-Verlag.

3. C. S. Hsu and W.-H. ChENG 1974 Transactions of the American Society of Mechanical Engineers, Journal of Applied Mechanics 371-378. Steady state response of dynamical system under combined parametric and forcing excitations.

4. A. Kahraman and R. Singh 1990 Journal of Sound and Vibration 142, 49-75. Nonlinear dynamics of a spur gear pair.

5. S. W. SHaW and P. J. Holmes 1983 Journal of Sound and Vibration 90, 129-155. A periodically forced piecewise linear oscillator.

6. S. NatSiavas 1989 Journal of Sound and Vibration 134, 315-331. Periodic response and stability of oscillators with symmetric trilinear restoring force.

7. G. R. Tomlinson and J. Lam 1984 Journal of Sound and Vibration 96, 111-125. Frequency response characteristics of structures with single and multiple clearance-type nonlinearity. 
8. F. Pfeiffer and A. Fritzer 1992 Z. angew. Math. Mech. 72, T38-T40. Resonanz und Tilgung bei spielbehafteten Systemen.

9. Y. S. CHOI and S. T. NoAH 1988 Journal of Sound and Vibration 121, 117-126. Forced periodic vibration of unsymmetric piecewise-linear systems.

10. Y. B. KIm and S. T. NoAH 1991 Transactions of the American Society of Mechanical Engineers, Journal of Applied Mechanics 58, 545-553. Stability and bifurcation analysis of oscillators with piecewise-linear characteristics: a general approach.

11. S. L. LAU and W.-S. ZHANG 1992 Transactions of the American Society of Mechanical Engineers, Journal of Applied Mechanics 59, 153-160. Nonlinear vibrations of piecewise linear systems by incremental harmonic balance method.

12. F. Pfeiffer and A. Kunert 1990 Nonlinear Dynamics 1, 63-74. Rattling models from deterministic to stochastic processes.

13. I. A. MAhfouz and F. BAdrakhan 1990 Journal of Sound and Vibration 143, 289-328. Chaotic behavior of some piecewise-linear systems, part II: systems with clearances.

14. A. Kahraman 1992 Nonlinear Dynamics 3, 183-198. On the response of a preloaded mechanical oscillator with a clearance: period doubling and chaos.

15. A. Kahraman and R. Singh 1991 Journal of Sound and Vibration 146, 135-156. Interactions between time-varying mesh stiffness and clearance non-linearities in a gear system.

16. K. Sato, S. Yamamoto and T. Kawakami 1991 Computational Mechanics 7, 173-182. Bifurcation sets and chaotic states of a gear system subjected to harmonic excitation.

17. S. F. MAsri, Y. A. Mariamy and J. C. Anderson 1981 Transactions of the American Society of Mechanical Engineers, Journal of Applied Mechanics 48, 404-410. Dynamic response of a beam with geometric nonlinearity.

18. F. C. Moon and S. W. Shaw 1983 International Journal of Non-linear Mechanics 18, 465-477. Chaotic vibrations of a beam with nonlinear boundary conditions.

19. S. W. SHAw 1985 Journal of Sound and Vibration 99, 199-212. Forced vibrations of a beam with one-sided amplitude constraint: theory and experiment.

20. P. V. BAYLy and L. N. VIRgIn 1993 Journal of Sound and Vibration 164, 364-374. An experimental study of an impacting pendulum.

21. N. Popplewell, C. N. Bapat and K. McLachlan 1983 Journal of Sound and Vibration 87, 41-59. Stable periodic vibroimpacts of an oscillator.

22. A. V. Oppenheim and R. W. Schafer 1975 Digital Signal Processing, Englewood Cliffs, New Jersey: Prentice-Hall.

23. R. A. Thisted 1988 Elements of Statistical Computing. New York: Chapman and Hall.

24. J. GuCKenHeIMER and P. Holmes 1983 Nonlinear Oscillations, Dynamical Systems and Bifurcations of Vector Fields. New York: Springer-Verlag.

25. A. Kahraman and G. W. BlanKenship 1995 Journal of Sound and Vibration in review. Interactions between commensurate parametric and external excitation in systems with clerance type non-linearity.

\section{APPENDIX A: ELEMENTS OF THE JACOBIAN MATRIX}

The elements of the Jacobian matrix $J_{i j}=\partial S_{i} / \partial u_{j}$ are given by

$$
\begin{gathered}
\frac{\partial S_{1}}{\partial u_{j}}=\frac{\partial v_{1}}{\partial u_{j}}+\frac{1}{2} \sum_{r=1}^{R}\left[w_{2 r}\left(\frac{\partial v_{2 r}}{\partial u_{j}}\right)+w_{2 r+1}\left(\frac{\partial v_{2 r+1}}{\partial u_{j}}\right)\right], \\
\frac{\partial S_{2 i}}{\partial u_{j}}=-(i \Lambda)^{2} \delta_{2 i}+2 i \Lambda \zeta \delta_{2 i+1}+w_{2 i}\left(\frac{\partial v_{1}}{\partial u_{j}}\right)+\left(\frac{\partial v_{2 i}}{\partial u_{j}}\right)+\frac{1}{2} \sum_{r=1}^{R}\left(\frac{\partial v_{2 r}}{\partial u_{j}}\right)\left[w_{2(r-i)}+w_{2(r+i)}+w_{2(i-r)}\right] \\
+\frac{1}{2} \sum_{r=1}^{R}\left(\frac{\partial v_{2 r+1}}{\partial u_{j}}\right)\left[w_{2(r-i)+1}+w_{2(r+i)+1}-w_{2(i-r)+1}\right], \quad i \in[1, R], \quad j \in[1,2 R+1],
\end{gathered}
$$




$$
\begin{aligned}
\frac{\partial S_{2 i+1}}{\partial u_{j}}= & -(i \Lambda)^{2} \delta_{2 i+1}-2 i \Lambda \zeta \delta_{2 i}+w_{2 i+1}\left(\frac{\partial v_{1}}{\partial u_{j}}\right)+\left(\frac{\partial v_{2 i+1}}{\partial u_{j}}\right) \\
& +\frac{1}{2} \sum_{r=1}^{R}\left(\frac{\partial v_{2 r}}{\partial u_{j}}\right)\left[-w_{2(r-i)+1}+w_{2(r+i)+1}+w_{2(i-r)+1}\right] \\
& +\frac{1}{2} \sum_{r=1}^{R}\left(\frac{\partial v_{2 r+1}}{\partial u_{j}}\right)\left[w_{2(r-i)}-w_{2(r+i)}+w_{2(i-r)}\right], \quad i \in[1, R], \quad j \in[1,2 R+1],
\end{aligned}
$$

where

$$
\delta_{i}= \begin{cases}1, & i=j, \\ 0, & i \neq j,\end{cases}
$$

is the Krohnecker delta. Meanwhile, the partial derivatives $\partial v_{i} / \partial u_{j}$ in equations (A1) are determined directly from equations (4) and (5) as [10]

$$
\begin{aligned}
& \frac{\partial v_{1}}{\partial u_{1}}=\frac{1}{N} \sum_{n=0}^{N-1} \phi_{n}, \\
& \frac{\partial v_{1}}{\partial u_{2 j}}=\frac{1}{N} \sum_{n=0}^{N-1} \phi_{n} \cos (2 \pi n j / N), \quad \frac{\partial v_{1}}{\partial u_{2 j+1}}=\frac{1}{N} \sum_{n=0}^{N-1} \phi_{n} \sin (2 \pi n j / N), \quad j \in[1, R], \\
& \frac{\partial v_{2 i}}{\partial u_{1}}=\frac{2}{N} \sum_{n=0}^{N-1} \phi_{n} \cos (2 \pi n i / N), \quad i \in[1, R] \\
& \frac{\partial v_{2 i}}{\partial u_{2 j}}=\frac{2}{N} \sum_{n=0}^{N-1} \phi_{n} \cos (2 \pi n i / N) \cos (2 \pi n j / N), \\
& \frac{\partial v_{2 i}}{\partial u_{2 j+1}}=\frac{2}{N} \sum_{n=0}^{N-1} \phi_{n} \cos (2 \pi n i / N) \sin (2 \pi n j / N), \quad i, j \in[1, R] \\
& \frac{\partial v_{2 i+1}}{\partial u_{1}}=\frac{2}{N} \sum_{n=0}^{N-1} \phi_{n} \sin (2 \pi n i / N), \quad i \in[1, R] \\
& \frac{\partial v_{2 i+1}}{\partial u_{2 j}}=\frac{2}{N} \sum_{n=0}^{N-1} \phi_{n} \sin (2 \pi n i / N) \cos (2 \pi n j / N), \\
& \frac{\partial v_{2 i+1}}{\partial u_{2 j+1}}=\frac{2}{N} \sum_{n=0}^{N-1} \phi_{n} \sin (2 \pi n i / N) \sin (2 \pi n j / N), \quad i, j \in[1, R],
\end{aligned}
$$

where $\phi_{n}$ is a discrete separation function given by

$$
\phi_{n}= \begin{cases}1, & \left|x_{n}\right|>1, \\ 0, & \left|x_{n}\right| \leqslant 1 .\end{cases}
$$

